\title{
A New Approach to Find Orthologous Proteins Using Sequence and Protein-Protein Interaction Similarity
}

\author{
Min Kyung Kim ${ }^{1}$, Young-Joo Seol ${ }^{2}$, Hyun Seok \\ Park, Seung-Hwan Jang ${ }^{1}$, Hang-Cheol Shin ${ }^{1}$ \\ and Kwang-Hwi Cho ${ }^{1 *}$
}

${ }^{1}$ Department of Bioinformatics and CAMDRC, Soongsil University, Seoul 156-743, Korea, ${ }^{2}$ Research Planning \& Information Div., National Institute of Agricultural Biotechnology, RDA, Suwon 441-707, Korea, ${ }^{3}$ Department of Computer Science, Ewha Womans University, Seoul 120-750, Korea

\begin{abstract}
Developed proteome-scale ortholog and paralog prediction methods are mainly based on sequence similarity. However, it is known that even the closest BLAST hit often does not mean the closest neighbor. For this reason, we added conserved interaction information to find orthologs. We propose a genome-scale, automated ortholog prediction method, named OrtholnterBlast. The method is based on both sequence and interaction similarity. When we applied this method to fly and yeast, $17 \%$ of the ortholog candidates were different compared with the results of Inparanoid. By adding protein-protein interaction information, proteins that have low sequence similarity still can be selected as orthologs, which can not be easily detected by sequence homology alone.
\end{abstract}

Keywords: interolog, ortholog, protein-protein interaction

\section{Introduction}

Ortholog is a term of evolution, which means that proteins have originated from the same ancestor protein but exist in different species, in contrast to paralogs in the same species (Koonin, 2005). They have the nature of sequence and function similarity. Therefore, finding the ortholog of certain proteins is important for further analysis of the protein (Chervitz et al., 1998).

Automated proteome-scale ortholog identification methods are categorized into BLASTp-based, phylogenybased, and evolutionary distance-based approaches (Chen et al., 2007). "Automated" means that there is no

*Corresponding author: E-mail chokh@ssu.ac.kr

Tel +82-2-820-0454, Fax +82-2-812-5762

Accepted 2 July 2009 human interference, which frequently happens in the phylogenetic tree construction method. BLASTp-based methods have recently developed and mainly depend on sequence similarity. Phylogeny- and evolutionary distance-based methods have also been suggested to overcome the lack of evolutionary information of the BLASTp-based approach-for example, Orthostrapper (Hollich et al., 2002), and RIO (Zmasek and Eddy, 2002).

COG (Tatusov et al., 2001) is designed for finding prokaryote orthologs, and KOG (Koonin et al., 2004), Inparanoid (Remm et al., 2001), and OrthoMCL (Li et al., 2003) are for eukaryotes. The basic idea of COG is the use of the selection of a 3-way reciprocal best match and protein sequence comparisons that are conducted by BLASTp (Altschul et al., 1990). Once the proteins are selected as orthologs in COG, they are excluded in the candidate gene pool. Therefore, when an ortholog is defined, it can not be replaced by other reasonable candidates, even if they have higher scores. Because COG selects orthologs using domain information, it is difficult to apply it to eukaryotes, which are abundant in multidomain proteins (Tatusov et al., 2001). For this reason, KOG has been developed for eukaryotes by the group who developed COG (Koonin et al., 2004). Inparanoid works by selecting orthologs by a reciprocal best hit, like COG. The method also considers the existence of paralogs. Therefore, Inparanoid defines ortholog relations that are sometimes one-to-many or many-to-many (Remm et al., 2001).

OrthoMCL (Li et al., 2003) is another kind of ortholog-finding system. This method can find orthologs among several species at the same time, which was impossible for other methods. OrthoMCL uses an allagainst-all BLAST search and a clustering algorithm based on the Markov model to select an ortholog group.

However, these approaches above are basically using BLAST to check sequence similarity, and the proteins that have the best BLAST score are selected as the main orthologs. It has been known that proteins that have the best similarity scores in BLAST search are often not the closest relatives phylogenetically (Koski and Golding, 2001). This implies that there is a possibility that genuine orthologs can not be found using sequence similarity alone.

Not only can sequence similarity be used for ortholog finding but also structure and interaction data. Even though the sequence similarity is not discovered, the 
proteins that have similar structures could conduct similar functions (Fribourg and Conti, 2003). Structural information, therefore, is an important key to classify the family and could be used to detect remote homologs. However, known protein structures are quite limited for use in genome-scale ortholog finding.

Alternatively, proteome-scale interaction data are available in $E$. coli, yeast, fly, worm, and human. It is known that proteins that have more interactors evolve more slowly (Fraser et al., 2002), interacting proteins co-evolve with their counterparts (Goh et al., 2000), and protein interfaces are more conserved than other surfaces (Caffrey et al., 2004). These findings are strong evidence of the relationship between evolution and protein-protein interaction data. The proteins that have conserved interactions are called "interologs" (Matthews et al., 2001; Yu et al., 2004). For this reason, we added a new criterion, "interaction data," to Inparanoid, which is a BLASTp-based ortholog finding system. The information of conserved protein-protein interactions is applied to identify functionally related proteins (Bandyopadhyay et al., 2006). However, they only analyze 121 cases of functional orthologs. In this paper, we suggest a genome-scale ortholog prediction system, OrtholnterBlast. In this method, not only sequence similarity but also protein-protein interaction data are used for finding orthologs across species. Comparison of the proteomscale ortholog prediction system is shown in Table 1.

\section{Methods}

OrtholnterBlast predicts the orthologs by the following steps; (1) choosing ortholog candidates based on sequence similarity, (2) comparing the interacting partner of the ortholog candidates, (3) scoring the sequence and interaction similarity by graph alignment, and (4) deciding orthologs according to their score. OrtholnterBlast consists of three different modules: a sequence score module, interaction score module, and resolve module. The architecture of OrtholnterBlast is shown in Fig. 1.

\section{Input data and Pre-processing}

OrtholnterBlast requires 4 types of files as inputs: protein ID, protein sequence, sequence similarity, and interaction data. Protein ID file consists of a SwissProt or TrEMBL ID and its related information, such as the protein name and description (Boeckmann et al., 2003). Except for the protein ID, other information is not essential. They can be used as additional information for verification only. The information of protein sequences came from Inparanoid. We used 18,932 protein sequences of fly (Drosophila melanogaster) and 6706 protein sequences of yeast (Saccharomyces cerevisiae). The third one is the BLAST result of fly-fly, fly-yeast, yeast-fly, and yeast-yeast. In OrtholnterBlast, a proteome-scale BLAST search should be performed 4 times to find orthologs of the two species, because a change

Table 1. Comparison of the proteome-scale ortholog prediction systems

\begin{tabular}{|c|c|c|c|}
\hline & OrthMCL & Inparanoid & OrtholnterBlast \\
\hline BLAST version & WU-BLAST & NCBI-BLAST & BLASTp \\
\hline BLAST Search & All-against-all & All-against-all & All-against-all \\
\hline \multirow[t]{2}{*}{ Similarity cutoff } & $\mathrm{p}<1 \mathrm{e}-5$ & Score $\geq 50$ bits & Overlap $>50 \%$ \\
\hline & & Score $\geq 50$ bits & Overlap $>50 \%{ }^{1}$ \\
\hline Reciprocal best hits & $\mathrm{p}$-value & Percent identity score & Percent identity score \\
\hline
\end{tabular}

${ }^{1}$ These options can be changed by the user in OrtholnterBlast.

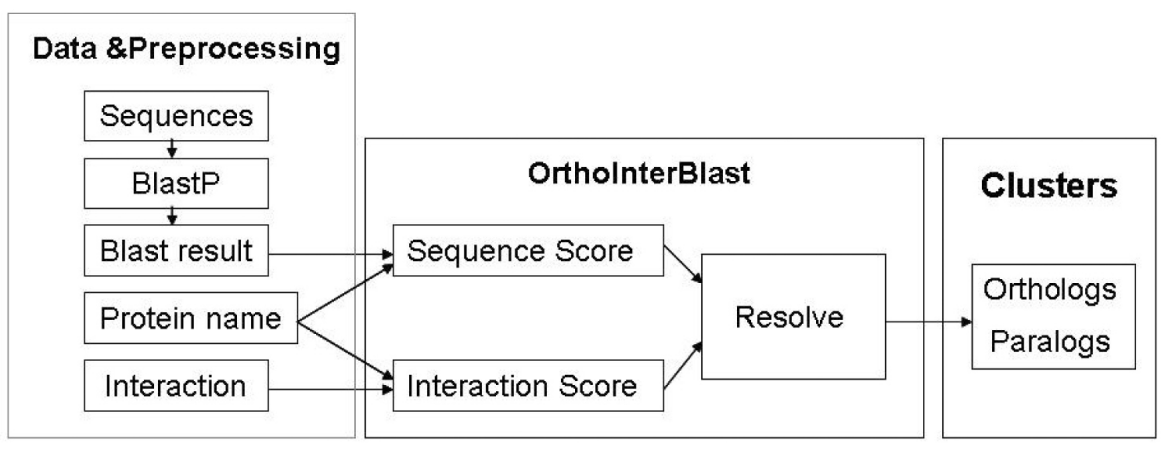

Fig. 1. System Architecture of OrtholnterBlast. 
of query and target in a BLAST search sometimes gives different results. Therefore, we compared fly-yeast and yeast-fly separately and used the mean value as a similarity score (Li et al., 2003).

If we conduct a BLAST search whenever OrtholnterBlast is running, the speed of the system will be decreased. Therefore, we conducted BLASTp prior to the main search and saved the result as an input file. The conditions for the BLASTp search were as follows: Grey zone is 0 bits, Score cutoff is 50 bits, In-paralogs, paralogs defined by Inparanoid, with confidence less than 0.05 , Sequence overlap cutoff is 0.05 , Group merging cutoff is 0.05 , and Scoring matrix is BLOSUM62 ( $\mathrm{Li}$ et al., 2003).

The result of BLASTp between different species, such as fly-yeast and yeast-fly, is used to find ortholog candidates. For example, $a^{\prime}$ and $a^{\prime \prime}$ show the similarity of $a$ and $a^{\prime}$, and $a^{\prime \prime}$ is the ortholog candidate in OrtholnterBlast. Then, the information is analyzed further by using the interaction score module for finding the main ortholog. The result of BLAST between the same species, such as fly-fly and yeast-yeast, will be used to find the paralog.

The last input data are the information of interacting partners. The information originates from the DIP (Database of Interacting Proteins) (Xenarios et al., 2002). The numbers of data used in OrtholnterBlast that are the overlap of both databases (DIP and Inparanoid) are 12,868 interactions among 4398 proteins and 18,197 interactions among 6628 proteins for yeast and fly, respectively (Table 2 ).

\section{OrtholnterBlast}

OrtholnterBlast consists of sequence, interaction, and resolve modules, as shown in Fig. 1. First, the sequence module selects ortholog candidates according to their sequence similarity. The threshold of sequence similarity score was set to the cutoff value. The current default value is 50 bit homology, as used in Inparanoid, and each candidate also has the sequence similarity score.

Table 2. The numbers of proteins and interactions used in OrtholnterBlast

\begin{tabular}{lrr}
\hline & \multicolumn{2}{c}{ Organism } \\
\cline { 2 - 3 } & \multicolumn{1}{c}{ Fly } & \multicolumn{1}{c}{ Yeast } \\
\hline No. of proteins from DIP & 7,052 & 4,749 \\
No. of proteins from Inparanoid & 18,931 & 6,705 \\
No. of interactions from DIP & 20,789 & 15,131 \\
No. of proteins from OrtholnterBlast & 6,628 & 4,398 \\
No. of interactions from OrtholnterBlast & 18,197 & 12,868 \\
\hline
\end{tabular}

Then, the candidates enter the interaction module. OrtholnterBlast performs a local graph alignment only for the candidates that have a sequence score above the cutoff value. The process of the local graph alignment is as follows: (1) If $A$ 's ortholog candidate is $a$, it generates new node $A a$. (2) A's interacting partner is $B$, $D$, and $F$ in yeast. Its ortholog candidates are $b, d$, and $f$, which are interacting with $a$ in fly. Then, the new nodes $B b, D d$, and $F f$ are generated by direct interaction with $A a$ in local graph alignment. (3) $c$ protein is related to $a$ directly, but $C$ protein is related to $A$ through $B$ (the distance between $C$ and $A$ is 2). This situation between $A a$ and $C c$ is defined as a "gap" in graph theory. The relation between $B b$ and $C c$ is the same. (4) The "mismatch" stands for the case that has the same distance through a different bridge node, as shown in the relation between $A a$ and $E e$ (Fig. 2).

Local graph alignment is similar to sequence alignment. "Direct interaction" in the graph is the same as "match" in the sequence alignment. "Gap" and "mismatch" are used terms in sequence alignment, too. These concepts are applied in PathBlast (Kelley et al., 2004) at the first time, which is aligning two different protein interaction networks to find conserved pathways. OrtholnterBlast follows the same graph alignment rule to find new ortholog candidates from the interaction network.

After the construction of a global network for each ortholog candidate, we were able to get the score of interaction similarity. We calculated the interaction similarity scores of each ortholog candidate according to the sum of the direct, gap, and mismatch interaction numbers with their weights. The weights were 10,7 ,

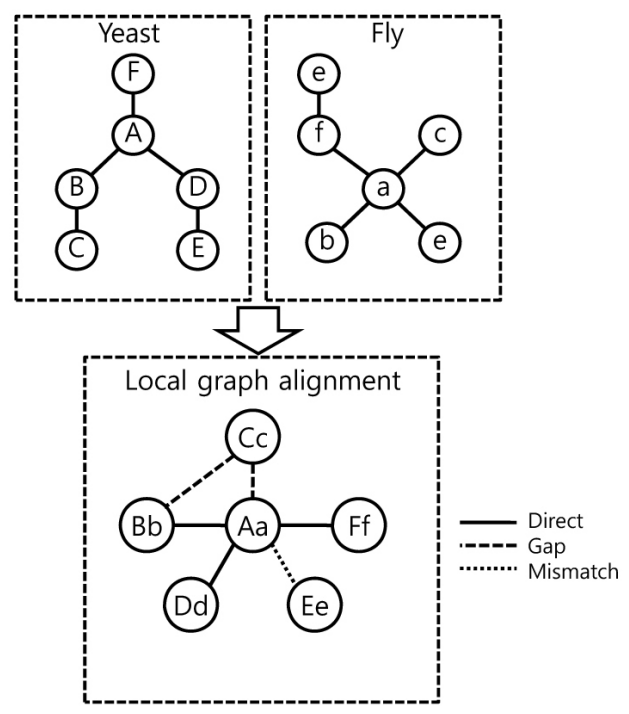

Fig. 2. Construction of local graph alignment. 
and 4 for the direct, gap, and mismatch interaction, respectively, which are used in the PathBlast scoring function. In case of Fig. 2, 3 direct interactions, 2 gaps, and 1 mismatch were found, so the total sum will be 48 $(3 \times 10+2 \times 7+1 \times 4=48)$. Then, the total sums are divided by the average number of interacting protein partners.

Finally, the sum of the calculated sequence and interaction similarity scores enters the resolve module as an input. Then, the resolve module defines orthologs and paralogs from the candidates by using their interaction and sequence similarity scores. The final decision of ortholog and paralog is made by the rules used in Inparanoid (Remm et al., 2001). The major difference from Inparanoid is that the score in OrtholnterBlast contains interaction similarity scores.

\section{Results}

OrtholnterBlast suggests 1922 ortholog groups between fly and yeast. Part of the results is shown in Fig. 3, and the full results are available in the supplementary materials. For further analysis, we compared the results with Inparanoid (Fig. 4) and summarized them in Table
3. An identical cluster should have the same ortholog and paralog pair. A non-identical cluster can be classified to mismatch and match groups. Match means that its cluster has the same ortholog protein but has a different paralog protein(s). Mismatch has a different ortholog protein pair but includes it as a paralog protein.

To find out the relative contributions of two pieces of information, sequence and interaction information, 2 sets of the ratio between sequence and interaction scores were tested (50:50, and 20:80). As the portion of the interaction score increased, the identical cluster with the result of Inparanoid decreased from $83 \%$ for $50: 50$ to $78 \%$ for $20: 80$. The number of clusters found with OrtholnterBlast decreased slightly compared to that of Inparanoid. Because proteins that are used in OrthoInterBlast should have the protein interaction information as well, the proteins without any interaction information were eliminated prior to the OrtholnterBlast calculation (As shown in Table 2).

For the case of 50:50, the number of identical clusters is 1622 out of 1963 (83\%). The $17 \%$ difference has been made by introducing the interaction score into OrtholnterBlast compared to Inparanoid. The trend for

\begin{tabular}{|c|c|}
\hline \multicolumn{2}{|c|}{ 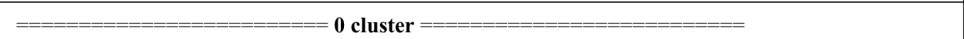 } \\
\hline \multicolumn{2}{|l|}{ RIR2_DROME : 1.0} \\
\hline \multirow{2}{*}{$\begin{array}{l}\text { DESC : Ribonucleoside diphosphate reductase } \\
\text { small subunit (CG8975-PA) }\end{array}$} & DESC : ribonucleoside-diphosphate reductase small \\
\hline & chain \\
\hline \multirow{2}{*}{$\begin{array}{l}\text { Total Score : } 68.35858585858585 \\
\text { seq Length : } 393.0\end{array}$} & Total Score : 68.35858585858585 \\
\hline & seq Length : 399.0 \\
\hline \multirow{2}{*}{$\begin{array}{l}\text { Seq Score : } 54.7979797979798 \% \\
\text { Inter Score : } 99.99999999999997 \%\end{array}$} & Seq Score : $54.7979797979798 \%$ \\
\hline & Inter Score : $99.99999999999997 \%$ \\
\hline \multirow{7}{*}{$\begin{array}{l}\text { Target protein: RIR2_YEAST } \\
\text {---interaction pairs---- } \\
\text { Q9VIT3:CG10364-PA open reading frame } \\
\text { Q9V8M7:CG15080-PA open reading frame } \\
\text { Q9V996:CG18584-PA open reading frame } \\
\text { Q9VCC9:CG6147-PA open reading frame } \\
\text { Q8SX59:CG9083-PA open reading frame }\end{array}$} & ||Target protein: RIR2_DROME \\
\hline & |---interaction pairs----- \\
\hline & ALG5_YEAST:dolichyl-phosphate \\
\hline & glucosyltransferase \\
\hline & RIR4_YEAST:ribonucleoside-diphosphate reductase \\
\hline & chain RNR4 \\
\hline & CYAA_YEAST:adenylate cyclase \\
\hline \multirow{13}{*}{ Q } & DBP8_YEAST:helicase homolog \\
\hline & RA10_YEAST:RAD10 protein \\
\hline & COPP_YEAST:coatomer complex beta' chain \\
\hline & SIR3_YEAST:regulatory protein SIR3 \\
\hline & PP11_YEAST:phosphoprotein phosphatase SIT4 \\
\hline & SMK1_YEAST:protein kinase SMK1 \\
\hline & TEM1_YEAST:GTP-binding protein TEMI \\
\hline & WTM2_YEAST:transcription modulator WTM2 \\
\hline & |YAH3_YEAST:FUN16 protein \\
\hline & Q04177:hypothetical protein YDR398w \\
\hline & ASF1_YEAST:ASF1 protein \\
\hline & P89501:nonhistone chromosomal protein NHP6B \\
\hline & \\
\hline
\end{tabular}

Fig. 3. Snapshot of OrtholnterBlast result. The ortholog pairs are shown in the first line of each cluster number. Protein function description, score, and interaction pair list are supplied as additional information. 


\begin{tabular}{|c|c|c|c|}
\hline \multicolumn{4}{|l|}{ identical } \\
\hline NAME:Q9VBU7 & NAME:Q9VBU7 & NAME:YRB1_YEAST & NAME:YRB1_YEAST \\
\hline ID:1904 & |ID:1390 & ID:1904 & ID:1390 \\
\hline Score: 1.993007 & Score: 114.0 & Score:1.993007 & Score: 114.0 \\
\hline Confidence: 1.0 & Confidence: 1.0 & Confidence: 1.0 & Confidence: 1.0 \\
\hline \multicolumn{4}{|c|}{--Match-- : OrthoInterBlast cluster:1903 Inparanoid cluster:1495 } \\
\hline NAME:Q8IH66 & NAME:Q8IH66 & NAME:YMH2_YEAST & NAME:YMH2_YEAST \\
\hline ID:1903 & ||ID:1495 & ||ID:1903 & |ID:1495 \\
\hline Score:2.3028786 & Score: 101.0 & Score:2.1389241 & Score: 101.0 \\
\hline Confidence: 1.0 & Confidence: 1.0 & Confidence: 1.0 & Confidence: 1.0 \\
\hline NAME:Q9VC62 & NAME:Q9VC62 & NAME:YNI7_YEAST & NAME:YNI7_YEAST \\
\hline ID:1903 & ID:1495 & || ID: 1903 & ID: 1495 \\
\hline Score:2.1389241 & Score: 101.0 & Score:2.3028786 & Score: 101.0 \\
\hline Confidence: 1.0 & Confidence: 1.0 & Confidence: 1.0 & \\
\hline NAME:Q8MRR8 & & NAME:Q12466 & \\
\hline ID: 1903 & & ID: 1903 & \\
\hline Score: 1.9977762 & & Score:1.9977762 & \\
\hline Confidence: 1.0 & & Confidence: 1.0 & \\
\hline \multicolumn{4}{|l|}{ NAME:Q9V4C4 } \\
\hline ID:1903 & & & \\
\hline Score: 1.4836112 & & & \\
\hline Confidence: 0.3518163 & & & \\
\hline
\end{tabular}

Fig. 4. Comparison of ortholog groups identified by OrtholnterBlast with those with Inparanoid (Each column indicates OrtholnterBlast Fly, Inparanoid fly, OrtholnterBlast yeast, Inparanoid yeast from left to right. This is a snapshot of OrthoInterBlast output).
20:80 is similar to that of $50: 50$ (Table 3).

We tried to verify the results in several ways. First, the EC number was used to evaluate the quality of the predicted orthologs with OrtholnterBlast. However, EC numbers are mostly based on sequence similarity and the groups in EC gene numbering system that are larger than the clusters in OrtholnterBlast and Inparanoid. Most of the EC numbers of the members in the clusters from both methods are the same, so it does not discriminate the superiority of our systems to Inparanoid. Second, structural similarity among orthologs has been considered. Unfortunately, structural information for yeast and fly are not enough to verify the result. As the structural information is increased, the results will be verified in the future.

Most of the methods so far have been developed based on sequence similarity, so it has a genuine limitation for finding remote homology that has very low sequence similarity. Even though it was not successful to find a proper way of validating our method, this method can overcome the genuine limitation of a sequence- only-based method, and the ability of the method could be improved as the interaction data increased. Also, global graph alignment without using sequence similarity would improve the predictability of OrtholnterBlast.
Table 3. Comparison of ortholog groups identified by OrtholnterBlast with Inparanoid

\begin{tabular}{|c|c|c|c|}
\hline \multicolumn{2}{|r|}{$50: 50^{1}$} & $\begin{array}{l}\text { No. of fly } \\
\text { proteins }\end{array}$ & $\begin{array}{l}\text { No. of yeast } \\
\text { proteins }\end{array}$ \\
\hline \multicolumn{2}{|c|}{ Clusters from OrtholnterBlast } & 3,398 & 2,368 \\
\hline \multicolumn{2}{|c|}{ Clusters from Inparanoid } & 3,792 & 2,473 \\
\hline \multicolumn{2}{|l|}{ Match } & 104 & 40 \\
\hline \multicolumn{2}{|l|}{ Mismatch } & 284 & 12 \\
\hline \multicolumn{2}{|r|}{$20: 80^{1}$} & $\begin{array}{l}\text { No. of fly } \\
\text { proteins }\end{array}$ & $\begin{array}{l}\text { No. of yeast } \\
\text { proteins }\end{array}$ \\
\hline \multirow{2}{*}{\multicolumn{2}{|c|}{$\begin{array}{l}\text { Clusters from OrtholnterBlast } \\
\text { Clusters from Inparanoid }\end{array}$}} & 3,185 & 2,239 \\
\hline & & 3,792 & 2,473 \\
\hline \multicolumn{2}{|l|}{ Match } & 98 & 38 \\
\hline \multicolumn{2}{|l|}{ Mismatch } & 247 & 16 \\
\hline Ratio & OrtholnterBlast & $\begin{array}{l}\text { Identical clusters } \\
\text { with Inparanoid }\end{array}$ & $\begin{array}{c}\text { Percent of } \\
\text { identical } \\
\text { clusters }^{2}\end{array}$ \\
\hline $50: 50^{1}$ & 1,922 & 1,622 & $83 \%$ \\
\hline $20: 80^{1}$ & 1,814 & 1,533 & $78 \%$ \\
\hline
\end{tabular}

${ }^{1}$ Ratio between the sequence similarity and interaction data used, ${ }^{2}$ Compared to Inparanoid. 


\section{Discussion}

One advantage of OrtholnterBlast is its expansibility to other groups of species when genomic-scale interaction data are available. We also applied it to $E$. coli, yeast, fly, C. elegans, mouse, and humans using OrtholnterBlast. The tendencies are also shown to have the same trend with fly-yeast (you can find these results at ebio.ssu. ac. kr/OIB )

The difference between Ideker's methods (Bandyopadhyay et al., 2006) and OrtholnterBlast is the usage of sequence information. Although sequence similarity decides the first ortholog candidate in Ideker's system and OrtholnterBlast, sequence score also contributes to the final decision of the ortholog in OrtholnterBlast. It is because we want to reduce the effect of false positive interaction data (Li et al., 2006).

We suggest a new system for finding ortholog proteins, OrtholnterBlast, based on sequence similarity and graph alignment. The result is compared to that of Inparanoid, and $17 \%$ of clusters are different between both methods. For verification, comparing the EC number shows as good a result as Inparanoid. Structural verification was not successful, because structural information for yeast and fly are very limited. However, including interaction data is very useful to find orthologs that have very low sequence similarity (remote homologous protein). As the quantity of interaction data increases, OrtholnterBlast can be a more powerful tool for functional ortholog finding.

\section{Acknowledgements}

This work was supported by a Korea Research Foundation Grant (KRF-2005-005-J01101).

\section{References}

Altschul, S.F., Gish, W., Miller, W., Myers, E.W., and Lipman, D.J. (1990). Basic local alignment search tool. J. Mol. Biol. 215, 403-410.

Bandyopadhyay, S., Sharan, R., and Ideker, T. (2006). Systematic identification of functional orthologs based on protein network comparison. Genome Res. 16, 428-435.

Boeckmann, B., Bairoch, A., Apweiler, R., Blatter, M.C., Estreicher, A., Gasteiger, E., Martin, M.J., Michoud, K., O'Donovan, C., and Phan, I. (2003). The SWISS-PROT protein knowledgebase and its supplement TrEMBL in 2003. Nucl. Acids Res. 31, 365-370.

Caffrey, D.R., Somaroo, S., Hughes, J.D., Mintseris, J., and Huang, E.S. (2004). Are protein-protein interfaces more conserved in sequence than the rest of the protein surface? Protein Sci. 13, 190-202.

Chen, F., Mackey, A.J., Vermunt, J.K., and Roos, D.S. (2007). Assessing performance of orthology detection strategies applied to eukaryotic genomes. PLOS One 2, e383.

Chervitz, S.A., Aravind, L., Sherlock, G., Ball, C.A., Koonin, E.V., Dwight, S. S., Harris, M.A., Dolinski, K., Mohr, S., and Smith, T. (1998). Comparison of the complete protein sets of worm and yeast: orthology and divergence. Science 282, 2022-2028.

Fraser, H.B., Hirsh, A.E., Steinmetz, L.M., Scharfe, C., and Feldman, M.W. (2002). Evolutionary rate in the protein interaction network. Science 296, 750-752.

Fribourg, S., Conti, E. (2003). Structural similarity in the absence of sequence homology of the messenger RNA export factors Mtr2 and p15. EMBO Rep. 4, 699-703.

Goh, C.S., Bogan, A.A., Joachimiak, M., Walther, D., and Cohen, F.E. (2000). Co-evolution of proteins with their interaction partners. J. Mol. Biol. 299, 283-293.

Hollich, V., Storm, C.E., and Sonnhammer, E.L. (2002). OrthoGUI: graphical presentation of Orthostrapper results. Bioinformatics 18, 1272-1273.

Kelley, B.P., Yuan, B., Lewitter, F., Sharan, R., Stockwell, B.R., and Ideker, T. (2004). PathBLAST: a tool for alignment of protein interaction networks. Nucl. Acids Res. 32, W83-W88.

Koonin, E.V., Fedorova, N.D., Jackson, J.D., Jacobs, A.R., Krylov, D.M., Makarova, K.S., Mazumder, R., Mekhedov, S.L., Nikolskaya, A.N., and Rao, B.S. (2004). A comprehensive evolutionary classification of proteins encoded in complete eukaryotic genomes. Genome Biol. 5, R7.

Koonin, E.V. (2005). Orthologs, paralogs, and evolutionary genomics. Annu. Rev. Genet. 39, 309-338.

Koski, L.B., and Golding, G.B. (2001). The closest BLAST hit is often not the nearest neighbor. J. Mol. Evol. 52, 540-542.

Li, L., Stoeckert, C.J.Jr., and Roos, D.S. (2003). OrthoMCL: Identification of Ortholog Groups for Eukaryotic Genomes. Genome Res. 13, 2178-2189.

Li, D., LI, J., Ouyang, S., Wang, J., Wu, S., Wan, P., Zhu, Y., Xu, X., and He, F. (2006). Protein interaction networks of saccharomyces cerevisiae, caenorhabditis elegans and drosophila melanogaster: large-scale organization and robustness. Proteomics 6, 456-461.

Matthews, L.R., Vaglio, P., Reboul, J., Ge, H., Davis, B.P., Garrels, J., Vincent, S., and Vidal, M. (2001). Identification of Potential Interaction Networks Using SequenceBased Searches for Conserved Protein-Protein Interactions or "Interologs". Genome Res. 11, 2120-2126.

Remm, M., Storm, C.E., and Sonnhammer, E.L. (2001). Automatic clustering of orthologs and in-paralogs from pairwise species comparisons. J. Mol. Biol. 314, 10411052.

Tatusov, R.L., Natale, D.A., Garkavtsev, I.V., Tatusova, T.A., Shankavaram, U.T., Rao, B.S., Kiryutin, B., Galperin, M.Y., Fedorova, N.D., and Koonin, E.V. (2001). The COG database: new developments in phylogenetic classification of proteins from complete genomes. Nucl. Acids Res. 29, 22-28.

Xenarios, I., Salwinski, L., Duan, X.J., Higney, P., Kim, S.M., and Eisenberg, D. (2002). DIP, the Database of Interacting Proteins: a research tool for studying cellular net- 
works of protein interactions. Nucl. Acids Res. 30, 303305.

Yu, H., Luscombe, N. M., Lu, H. X., Zhu, X., Xia, Y., Han, J.D., Bertin, N., Chung, S., Vidal, M., and Gerstein, M. (2004). Annotation transfer between genomes: protein- protein interologs and protein-DNA regulogs. Genome Res. 14, 1107-1118.

Zmasek, C.M., and Eddy, S.R. (2002). RIO: analyzing proteomes by automated phylogenomics using resampled inference of orthologs. BMC Bioinformatics 3, 14. 